\title{
Organisation du système racinaire du chêne pédonculé (Quercus robur) développé en conditions édaphiques non contraignantes (sol brun lessivé colluvial)
}

\author{
E Lucot, S Bruckert * \\ Université de Franche Comté, laboratoire de pédologie, Place Leclerc, 25030 Besançon, France
}

(Reçu le 17 janvier 1992; accepté le 25 mai 1992)

\begin{abstract}
Résumé - La densité racinaire, l'inclinaison, l'extension latérale et en profondeur des racines ont été mesurées sur des chênes pédonculès âgés de 150 ans et établis dans un sol brun lessivé colluvial. Les matériaux pédologiques, limono-argileux puis argilo-limoneux, ont été étudiés en parallèle. Ils sont accumulés sur $4 \mathrm{~m}$ d'épaisseur au-dessus d'une roche calcaire karstifiée. Le sol actuel, ainsi que les horizons pédogénéisés sous-jacents, permettent un développement racinaire sans contrainte physique ou chimique, jusqu'à la roche. Les chênes pédonculés ont un système racinaire qui peut être divisé en 2 parties : le système de surface, qui s'étend jusqu'à $60 \mathrm{~cm}$ de profondeur et le système profond, situé en dessous de $60 \mathrm{~cm}$. Au niveau du système de surface et dans un rayon de $3 \mathrm{~m}$ autour de l'arbre, l'enracinement est intensif et composé de racines de tous diamètres (moins de $1 \mathrm{~mm}$ à plus de $10 \mathrm{~cm}$ ), avec une densité racinaire maximale. Les racines ont une inclinaison de $80-85^{\circ}$ par rapport à la verticale. L'enracinement extensif peut s'étendre jusqu'à une distance de $20 \mathrm{~m}$ de l'arbre. Le système racinaire profond, qui se développe dans un rayon de $2-2,5 \mathrm{~m}$, est subdivisé en 2 parties : de 60 à $120 \mathrm{~cm}$ (système profond intensif) et en dessous de $120 \mathrm{~cm}$, jusqu'à plus de $4 \mathrm{~m}$ (système profond extensif). II est composé de racines subverticales (pivots). Une estimation des volumes de sol prospectés de façon intensive et extensive donne respectivement 17 et $800 \mathrm{~m}^{3}$.
\end{abstract}

chêne pédonculé / relation racines-sol / système racinaire / paramètre racinaire / propriété physique du sol / sol brun lessivé colluvial

Summary - Common oak (Quercus robur) root system organisation developed without restricting edaphic conditions (colluvial leached brown soil). The root density, slope, and the lateral and depth extension of root spreading have been measured in 150-yr old common oaks (Quercus robur) growing on colluvial leached brown soil. A parallel study was also performed on the clayey silt then silty clay soil materials, which had accumulated to a depth of $4 \mathrm{~m}$ above karstified calcareous rock. The soil and the underlying layers permitted the root system to develop without any physical or chemical constraints as far as the rock. The root system of common oak can be divided into 2 parts: the surface system which spreads between $0-60 \mathrm{~cm}$ in depth; and the deep system below $60 \mathrm{~cm}$. In a 3-m radius around the tree at the surface system level rooting was intensive and consisted of roots of varying diameters (from $<1 \mathrm{~mm}$ to $>10 \mathrm{~cm}$ ), with a maximum root density. Roots sloped at an angle of $80-85{ }^{\circ} \mathrm{C}$ with respect to the vertical. The extensive rooting could

* Correspondance et tirés à part 
spread up to $20 \mathrm{~m}$ from the tree. The deep system spread 2-2.5 $\mathrm{m}$ and was composed of subvertical roots (tap roots). It can be divided in 2 parts: the intensive deep system, from $60-120 \mathrm{~cm}$; and the extensive deep system, from $120 \mathrm{~cm}$ to $>400 \mathrm{~cm}$. The volumes of the intensive and extensive soil examined were estimated at 17 and $800 \mathrm{~m}^{3}$ respectively.

common oak = Quercus robur / root-soil relation / root system / root parameter / soil physical property / colluvial leached brown soil

\section{INTRODUCTION}

L'appréciation de la fertilité d'un sol forestier repose sur l'observation des paramètres qui conditionnent la disponibilité en eau et en éléments minéraux pour les végétaux (Pritchett, 1979). Les critères physico-chimiques et phytosociologiques, en particulier dans le cas des stations mésotrophes, ne permettent pas à eux seuls d'apprécier et d'expliquer la fertilité des sols forestiers. Des éléments explicatifs doivent être recherchés dans les relations racine-sol (Callot, 1983). Le développement du système racinaire exerce en effet une influence prépondérante sur l'alimention hydrique et minérale (Forristall et Gessel, 1955; Lévy, 1968), notamment sur le réapprovisionnement de la rhizosphère et le déroulement de fonctions métaboliques qui limitent la croissance des plantes (Martens, 1975). Par conséquent, les niveaux de fertilité sont commandés par les possibilités de colonisation du sol par les racines et dépendent de critères morphoédaphiques inscrits, d'une part dans la morphologie des profils pédologiques jusqu'à l'interface sol-roche et, d'autre part, dans les relations qui s'établissent entre les végétaux et le sol.

La morphologie du système racinaire d'un arbre est fixée génétiquement, mais est susceptible de variations selon les facteurs de l'environnement (Weller, 1965; Vartanian, 1974; Pritchett, 1979, Henderson et al, 1983; Coutts, 1989). L'étude quantitative de l'enracinement et en particulier de sa distribution verticale et hori- zontale renseigne sur les possibilités de colonisation du sol par les racines et sur l'influence des contraintes physiques ou chimiques rencontrées. Pour définir et quantifier l'impact de ces facteurs, principalement pédologiques (Lucot, 1990), il est cependant indispensable de connaître les caractéristiques du système racinaire en conditions non contraignantes. C'est l'objectif que les travaux présentés ici ont cherché à atteindre. Pour cela, nous avons choisi une futaie de chênes pédonculés adultes de très bonne qualité (Mourey, 1979) situés dans leur station optimale mésohygrophile (Rameau et al, 1989) et en conditions édaphiques non contraignantes - milieu poreux et aéré des sols bruns lessivés colluviaux. Les études ont porté à la fois sur les systèmes racinaires et sur les propriétés des matériaux pédologiques colonisés par les racines.

\section{MATÉRIEL ET MÉTHODES}

\section{Caractéristiques des sites}

La station forestière choisie pour l'étude de l'enracinement des chênes pédonculés est située à $10 \mathrm{~km}$ au sud de Besançon, sur le plateau de Montrond-le-Château, à $355 \mathrm{~m}$ d'altitude (coordonnées Lambert $x=879,3 ; y=249,1$ ); elle occupe 20 ha dans le massif du Bois Nouveau (100 ha), sur le territoire communal de Fontain. La situation géomorphologique est celle d'un large vallon karstique drainé à la fois par des eaux souterraines et par un ruisseau temporaire. De courtes périodes d'inondation surviennent régulièrement en hiver (10 j en janvier 
1991). À la fin de leur séjour, les eaux sont évacuées très rapidement, en quelques heures, par les conduits karstiques. Les ruissellements ont déposé environ $4 \mathrm{~m}$ de matériaux würmiens (datation en cours) essentiellement limono-argileux, colluviaux et alluviaux, sur des calcaires sublithographiques du Bathonien (J2), fracturés et karstifiés.

Le site reçoit environ $1100 \mathrm{~mm}$ de précipitations bien réparties sur l'année. La température moyenne annuelle est de $10^{\circ} \mathrm{C}$. Compte tenu de l'évapotranspiration, un léger déficit hydrique climatique survient en juillet-août, mais ce demier est compensé par la réserve utile en eau du sol (125 et $206 \mathrm{~mm}$ à 60 à $100 \mathrm{~cm}$ de profondeur).

Le peuplement forestier est une futaie de chênes pédonculés âgés de 150 ans comportant un sous-étage de charme et une strate herbacée indiquant un niveau mésoneutrophile hygrocline, composée de Carex sylvatica, Anemone nemorosa, Arum maculatum, Glechoma hederacea, Ajuga reptens, Paris quadrifolia, Athyrium filix-fomina, Deschampsia cespitosa, Filipendula ulmaria et Valeriana officinalis subsp collina. Cette station est réputée pour la qualité de ses chênes, parmi les meilleurs de la région (Mourey, 1979). Leur étude a mis en évidence une qualité technologique très exceptionnelle, caractérisée par la rareté des fibres et une limite bois de printemps-bois d'été peu visible. Les tiges de parcelles exploitées depuis 1978 sur le site d'étude ont atteint les plus hauts prix lors des ventes régionales.

\section{Méthode d'étude des matériaux pédologiques}

Les matériaux pédologiques ont été observés en prenant comme référence le glossaire FAOUNESCO (1989) et la classification française (CPCS, 1967; Duchaufour, 1991). Un complément d'information portant sur l'organisation des horizons a été obtenu par une étude microscopique effectuée sur des sections fines $(6 \times 8 \mathrm{~cm})$ de prélèvements pris à $70,90,130,150,210$, $230,250,270,290,390$ et $410 \mathrm{~cm}$ de profondeur, consolidés par une résine de type araldite et préparées au centre de pédologie biologique du CNRS à Nancy.

Des échantillons de sol en vue d'analyse ont été prélevés tous les $20 \mathrm{~cm}$ selon des axes verticaux espacés de $2 \mathrm{~m}$ dans chaque fosse :
2 axes dans l'une, 3 dans l'autre. Sur les échantillons secs à l'air et tamisés à $2 \mathrm{~mm}$, on a déterminé la texture par la méthode internationale $\left(\mathrm{H}_{2} \mathrm{O}_{2}\right.$, héxamétaphosphate, pipette), le $\mathrm{pH}$ (eau/sol = 2,5/), la capacité d'échange cationique par la méthode à l'acétate d'ammonium, les cations $\mathrm{Ca}, \mathrm{Mg}, \mathrm{K}$, échangeables par absorption atomique, le $\mathrm{C}$ organique et minéral par dosage du $\mathrm{CO}_{2}$ au carmographe Whostoff. La densité apparente a été mesurée en prélevant des volumes connus d'échantillons qui ont été séchés à $105^{\circ} \mathrm{C}$ et pesés (5 répétitions). La porosité totale a été calculée à partir de la densité réelle $D r$ $(D r=2,65-0,015 \times C(\%))$ et de la densité apparente $D a$, par la formule : $P(\%)=(1-D a / D r) \times$ 100. La perméabilité a été estimée selon la méthode de McKeague et al (1982), basée sur l'observation de la porosité.

\section{Méthode d'étude de l'enracinement}

Dans une parcelle destinée à être exploitée au printemps 1991, avec un peuplement d'arbres adultes et vigoureux (55 arbres/ha), on a étudié l'organisation du système racinaire de chênes pédonculés.

À la base du tronc de 12 arbres choisis au hasard dans la station, on a dénombré les contreforts et les racines de diamètre supérieur à $50 \mathrm{~mm}$, ainsi que leurs ramifications dans un rayon de $1 \mathrm{~m}$ autour de l'arbre.

Sur 2 chênes pédonculés dominants dont les caractéristiques dendrométriques sont données dans le tableau I, on a déterminé les profils raci-

Tableau I. Caractéristiques dendrométriques des chênes de Fontain.

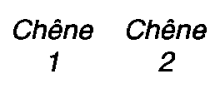

$\begin{array}{lcc}\text { Âge } & 150 & 145 \\ \text { Diamètre à 1,30 } \mathrm{m}(\mathrm{cm}) & 80 & 95 \\ \text { Hauteur totale }(\mathrm{m}) & 27 & 28 \\ \text { Longueur à la découpe }(\mathrm{m}) & 14,3 & 16,7 \\ \text { Diamètre médian }(\mathrm{cm}) & 71 & 80 \\ \text { Volume à la découpe }\left(\mathrm{m}^{3}\right) & 5,5 & 7,2 \\ \text { Rayon maximal du houppier }(\mathrm{m}) & 8,5 & 7,1\end{array}$


naires (Belgrand, 1983) le long d'une coupe verticale partant depuis un arc de cercle distant de $1 \mathrm{~m}$ du tronc, dans le prolongement d'un contrefort. Pour cela, 2 fosses de $3 \mathrm{~m} \times 4,5 \mathrm{~m}$ et $4,2 \mathrm{~m}$ de profondeur ont été creusées à la pelle mécanique à proximité de chaque chêne (distance entre les fosses : $60 \mathrm{~m}$ ).

Dans chaque fosse, un échafaudage a été placé le long de la face verticale la plus proche de l'arbre (fig 1A) de façon à aménager dans de bonnes conditions le profil cylindrique de $1,1 \mathrm{~m}$ nécessaire pour étudier le système racinaire (1/ 6 de celui de l'arbre). Pour chaque couche de $10 \mathrm{~cm}$ d'épaisseur, les racines ont été comptées par classe de diamètre $(1-5 \mathrm{~mm}$ : classe $a ; 5-20$ $\mathrm{mm}$ : classe b; 20-50 mm : classe c; $>50 \mathrm{~mm}$ : classe d). Lorsque la section d'une racine était répartie sur plusieurs niveaux, on a pris en compte séparément la fraction de racine appartenant à chaque niveau (fig 1B).

Le dénombrement des racines par classe de diamètre est exprimé en surface de racines sur la base du diamètre moyen de chaque classe et du diamètre réel pour la classe $d$. Les valeurs de surface pour chaque classe sont cumulées par tranche de sol de $10 \mathrm{~cm}$, de même pour l'ensemble du profil, puis exprimées en densité racinaire $\left(\mathrm{cm}^{2}\right.$ de racine/ $\mathrm{dm}^{2}$ de profil). Cette densité est rapportée à la surface cumulée totale du profil (toutes classes confondues) et exprimée en $\%$.

Ce travail ne permet pas l'accès aux racines qui ne recoupent pas le profil étudié et en particulier aux pivots (racines dont la direction fait un angle inférieur à $45^{\circ}$ avec un axe vertical). Les pivots ont été dégagés manuellement sur $80 \mathrm{~cm}$ en direction du centre de l'arbre, à partir du profil réalisé précédemment. La profondeur atteinte à leur ramification principale a été mesurée.

Les mesures concernant la répartition verticale et la profondeur de ramification ont été complétées par la mesure de l'inclination des racines de diamètre $>10 \mathrm{~mm}$ par rapport à la verticale.

L'extension latérale des racines du système racinaire profond (Sutton et Tinus, 1983) a été observée lors de l'ouverture de la fosse et dans celle-ci, à proximité du profil. L'extension du système de surface a été mesurée en dégageant les grosses racines depuis les troncs (fig 1D). Les coordonnées des racines ont été notées dans les 3 dimensions, dans un repère orthogonal : la profondeur $(Y)$ par rapport à la surface du sol, la distance au centre du tronc $(X)$ et l'écart de la racine par rapport à $X(Z)$. Les coordonnées sont mesurées tous les $0,50 \mathrm{~m}$ sur l'axe $X$.

\section{RÉSULTATS}

\section{Caractéristiques des matériaux pédologiques}

\section{Organisation}

Les caractéristiques morphologiques observées dans les profils et les sections fines (tableau II) ont montré que les $4 \mathrm{~m}$ de dépôts qui recouvrent la roche calcaire karstifièe, sont fortement structurés en polyèdres et en agrégats résultants de processus physiques de gonflement-retrait et de processus biologiques. Tous les niveaux colorés de façon homogène en brun jaunâtre $(10 \mathrm{YR})$ et brun rougeâtre $(7,5$ YR) apparaissent soumis à un pédoenvironnement aéré. Trois horizons présentent les caractères d'organisation en polyèdres revêtus d'argilanes des Bt argiliques et indiquent que 3 phases de pédogénèse apparentées au lessivage se sont succédées.

\section{Caractéristiques physiques et chimiques}

Les figures 2 et 3 renseignent sur les taux moyens des fractions granulométriques et la teneur en fer, avec la déviation standard à la moyenne au seuil de $0,01 \%$ obtenus à partir de 5 profils analysés.

Les horizons $A 1(0-10 \mathrm{~cm})$ et $E$ jusqu'à $60 \mathrm{~cm}$ sont limono-argileux. La base de l'horizon $E(60-80 \mathrm{~cm})$ et l'horizon $\mathrm{Bt}$ jusqu'à $180 \mathrm{~cm}$ sont argilo-limoneux. La teneur maximale en argile du $\mathrm{Bt}$ atteint $35 \%$ entre 100 et $120 \mathrm{~cm}$. L'indice de lessivage 


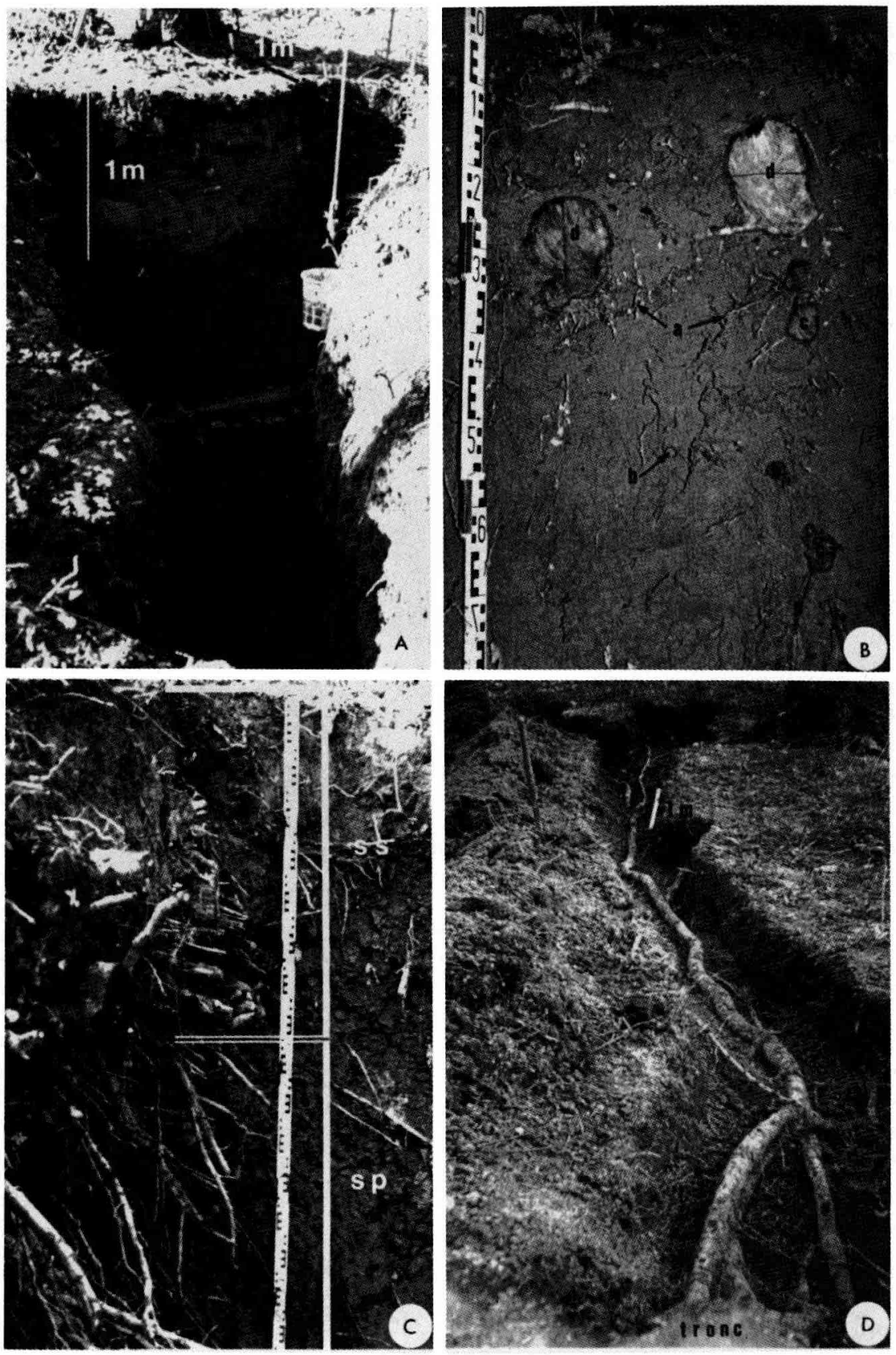

Fig 1. A. Disposition du profil en arc de cercle (à $1 \mathrm{~m}$ de distance de l'arbre) utilisé pour la mesure des racines; on aperçoit l'échafaudage permettant l'accès à la partie supérieure du profil. B. Sections de racines de tous diamètres ( $a, b, c, d)$ apparaissant au niveau du profil à $1 \mathrm{~m}$ de l'arbre, dans le système de surface; on a dégagé un pivot $(p)$ émis par une racine de classe $c$. C. Vue latérale du système racinaire à $1 \mathrm{~m}$ de l'arbre où l'on distingue les racines du système de surface (SS) subhorizontales et le système profond (SP) avec ses pivots presque verticaux. D. Parcours d'une grosse racine extensive du système de surface dégagée sur toute sa longueur. Seulement $1 / 4$ de son parcours, soit $6 \mathrm{~m}$, est visible sur cette vue. 
Tableau II. Caractéristiques morphologiques des matériaux pédologiques et de la roche sous-jacente de calcaire jurassique $\left(\mathrm{J}^{2}\right)$.

\begin{tabular}{|c|c|c|c|c|c|c|}
\hline \multirow{2}{*}{$\begin{array}{l}\text { Horizon } \\
\text { profondeur } \\
(\mathrm{cm})\end{array}$} & \multirow{2}{*}{$\begin{array}{l}\text { Texture } \\
\text { (USDA) }\end{array}$} & \multirow{2}{*}{$\begin{array}{l}\text { Couleur } \\
\text { (état sec) }\end{array}$} & \multicolumn{2}{|c|}{ Structure } & \multirow{2}{*}{$\begin{array}{c}\text { Argilanes } \\
-(\text { section fine })\end{array}$} & \multirow[t]{2}{*}{ Carbonates } \\
\hline & & & primaire $s$ & sous structure & & \\
\hline $\begin{array}{c}A 1 \\
0-20 \\
E \\
20-80\end{array}$ & $\begin{array}{l}\text { limoneuse } \\
\text { limono- } \\
\text { argileuse }\end{array}$ & $\begin{array}{l}10 \text { YR } 3 / 2 \\
2,5 \text { YR } 5 / 4\end{array}$ & $\begin{array}{l}\text { agrégats fins } \\
\text { agrégats fins }\end{array}$ & & & \\
\hline $\begin{array}{c}\mathrm{Bt} \\
80-160\end{array}$ & $\begin{array}{l}\text { argilo- } \\
\text { limoneuse }\end{array}$ & 10 YR $5 / 3$ & $\begin{array}{l}\text { polyèdrique } \\
\text { fines unités } \\
\text { très anguleuses }\end{array}$ & & $\begin{array}{c}\text { nombreux } \\
\text { bruns foncés }\end{array}$ & \\
\hline $\begin{array}{c}B C \\
160-220\end{array}$ & $\begin{array}{l}\text { argilo-limoneuse } \\
\text { avec sables } \\
\text { blancs }\end{array}$ & 10 YR 5/4 & columnaire fine & & & \\
\hline $\begin{array}{c}11 B C \\
220-280\end{array}$ & $\begin{array}{l}\text { limon-argilo- } \\
\text { sableuse }\end{array}$ & 10 YR $5 / 3$ & $\begin{array}{l}\text { prismatique } \\
\text { grossière }\end{array}$ & $\begin{array}{c}\text { prismatique } \\
\text { moyenne }\end{array}$ & & $\begin{array}{l}\text { légèrement } \\
\text { carbonaté }\end{array}$ \\
\hline $\begin{array}{c}\text { III Bt } \\
280-360\end{array}$ & argilo-limoneuse & 10 YR $5 / 8$ & $\begin{array}{c}\text { prismatique } \\
\text { grossière }\end{array}$ & littée fine & nombreux & \\
\hline $\begin{array}{c}\text { IV BC } \\
360-400\end{array}$ & argilo-limoneuse & 10 YR $6 / 8$ & litée fine & & & \\
\hline $\begin{array}{c}V B t \\
400-420\end{array}$ & argilo-limoneuse & 7.5 YR 5/6 & $\begin{array}{l}\text { polyèdrique } \\
\text { fine }\end{array}$ & & nombreux & \\
\hline $\mathbf{R}$ & roche calcaire bl & he, sublit & raphique, fra & ée et kar & ée & \\
\hline
\end{tabular}

est d'environ 1,7. Le fer accompagne les argiles, l'indice d'entraînement étant rigoureusement le même. La texture redevient limono-argileuse dans I'horizon BC de 180 à $220 \mathrm{~cm}$.

La texture change fortement, de même que la teneur en fer, dans I'horizon II BC $(220-280 \mathrm{~cm})$. Les sables attestent de l'origine alluviale de ce dépôt.

L'horizon III Bt $(260-360 \mathrm{~cm})$ est argilolimoneux, de même que l'horizon IV BC $(360-380 \mathrm{~cm})$ mais ce dernier renferme plus de fer que l'horizon III Bt. De 380 à $420 \mathrm{~cm}$, un horizon $\mathrm{V}$ Bt argileux rouge termine les matériaux d'origine pédologique, à l'interface avec la roche karstifiée.

La densité apparente augmente régulièrement entre la surface $\left(0,87 \mathrm{~g} \cdot \mathrm{cm}^{-3}\right)$ et $100 \mathrm{~cm}\left(1,44 \mathrm{~g} . \mathrm{cm}^{-3}\right)$; sa valeur moyenne pour les horizons situés au-dessous de $100 \mathrm{~cm}$ est de 1,49 $\pm 0,06$ g.cm ${ }^{-3}$. Le maximum $\left(1,58 \mathrm{~g} . \mathrm{cm}^{-3}\right)$ est atteint dans I'horizon limono-argilo-sableux II BC (220$240 \mathrm{~cm}$ ). La porosité totale, élevée dans les 40 premiers $\mathrm{cm}$ de profondeur, varie peu le long des profils (fig 4) : sa valeur moyenne est de $44,5 \pm 2,79 \%$. Les intervalles de confiance au seuil de $0,01 \%$ sont 

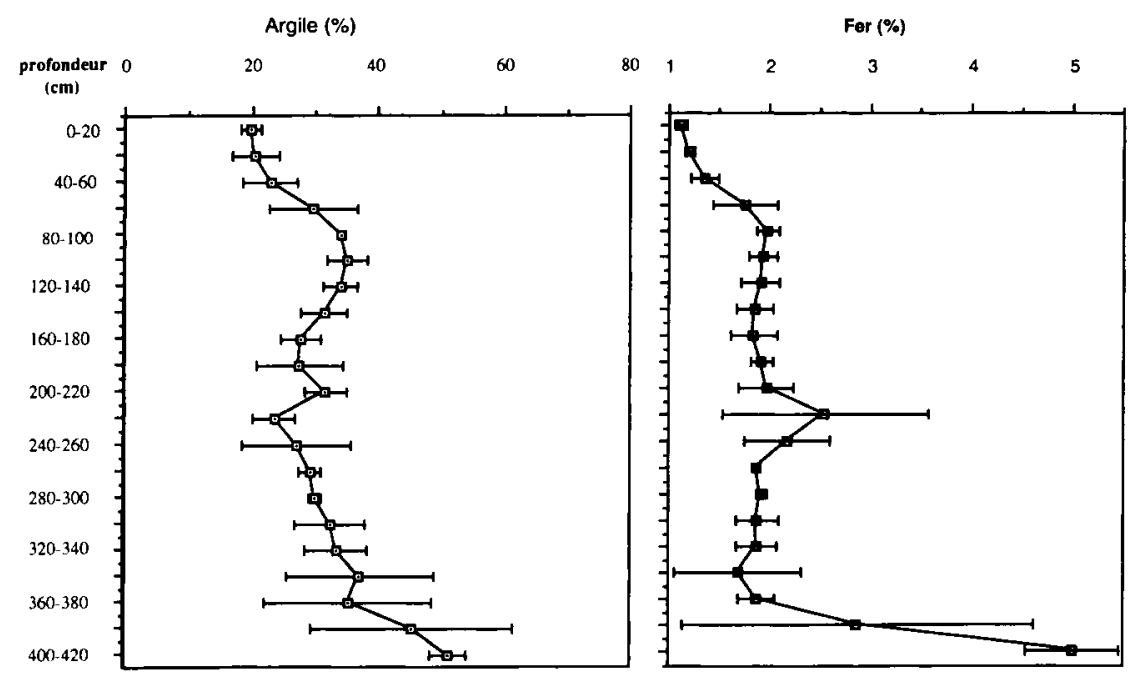

Fig 2. Variation moyenne des teneurs en argile et en fer sur 5 séries d'analyses avec la déviation standart à la moyenne au seuil de $0,01 \%$.

Teneurs $(\%)$

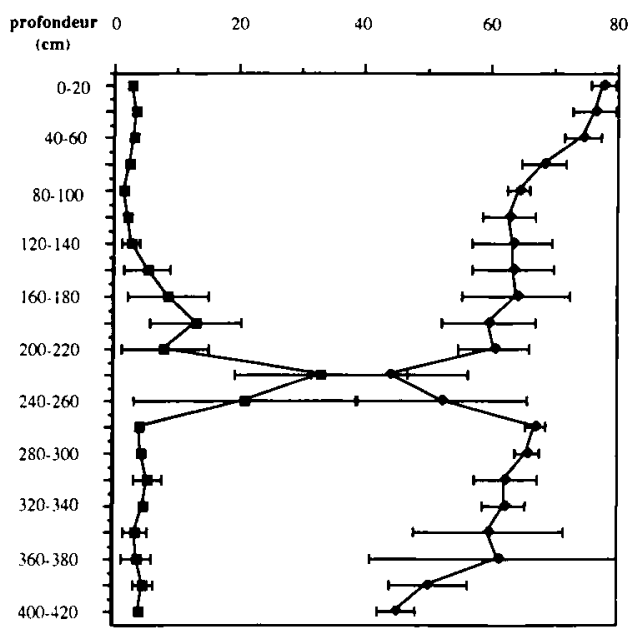

Fig 3. Variation moyenne des teneurs en limons $(\longmapsto-1)$ et en sables $(\longmapsto-1)$ sur 5 séries d'analyses avec la déviation standart à la moyenne au seuil de $0,01 \%$. porosite $(\%)$

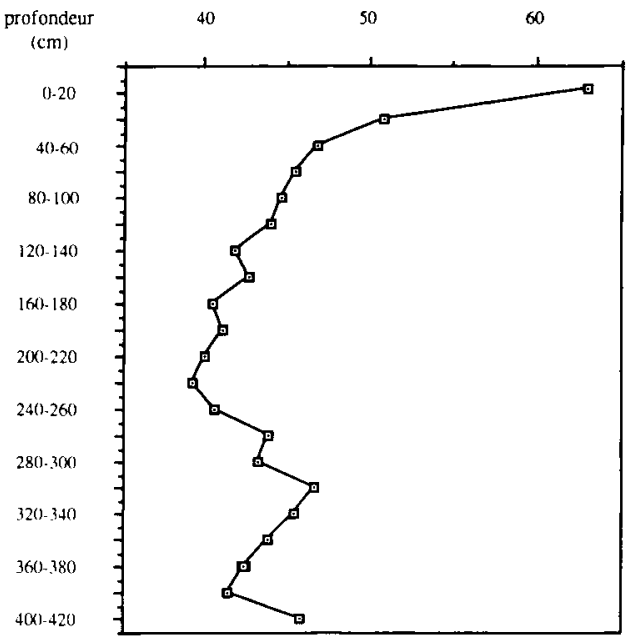

Fig 4. Variation moyenne de porosité ( en fonction de la profondeur, la déviation standart à la moyenne au seuil de $0,01 \%$ est trop faible pour être représentée. 
très faibles dans les 5 profils analysés. La perméabilité des horizons $A_{1}, A_{2}$ et $B t$ ( 0 $160 \mathrm{~cm}$ ) est forte $(K \mathrm{~s}>16,7 \mathrm{~cm} / \mathrm{h}$ ), puis plus faible $(1,7-4,2 \mathrm{~cm} / \mathrm{h})$ au niveau de I'horizon BC (160-220 cm), pour augmenter ensuite à partir de $220 \mathrm{~cm}$, la perméabilité étant moyenne $(4,2-16,7 \mathrm{~cm} / \mathrm{h})$ jusqu'à la roche.

Le profil de la capacité d'échange cationique suit les variations des taux d'argile, à l'exception des 60 premiers $\mathrm{cm}$ dans lesquels les charges de la matière organique s'ajoutent à celle de l'argile. La CEC moyenne atteint $14,1 \pm 2,7 \mathrm{meq} / 100 \mathrm{~g}$. Le profil est marqué par 2 valeurs minimales de $10,4 \mathrm{meq} / 100 \mathrm{~g}$ entre $40-60$ et $220-240$ $\mathrm{cm}$. Les rapports CEC/taux d'argile (en dehors des horizons de surface) sont sensiblement les mêmes et traduisent bien les différentes pédogénèses à partir de matériaux semblables.

Le sol contient $10 \mathrm{meq} / 100 \mathrm{~g}$ de calcium en surface, entre 7 et $8 \mathrm{meq} / 100 \mathrm{~g}$ jusqu'à $60 \mathrm{~cm}$. La teneur augmente ensuite et sature la CEC à partir de $80 \mathrm{~cm}$. La teneur en magnésium varie peu, sa moyenne étant de 0,28 $\pm 0,09 \mathrm{meq} / 100 \mathrm{~g}$ ). Le maximum se situe en surface : 0,67 meq/100 g. Il en est de même pour la teneur en potassium $(0,33 \pm 0,09 \mathrm{meq} / 100$ g) dont le maximum se situe aussi en surface : $0,55 \mathrm{meq} / 100 \mathrm{~g}$.
Les horizons lessivés jusqu'à $60 \mathrm{~cm}$ sont partiellement désaturés et mésotrophes (minimum de $56,6 \%$ entre 10 et 20 $\mathrm{cm}$ ); au-dessous de $60-80 \mathrm{~cm}$, le complexe d'échange devient saturé en bases. Le $\mathrm{pH}$ croît avec la profondeur, il est de 5,4 en surface, supérieur à 6,0 à $80 \mathrm{~cm}$ et dépasse 7,0 à $200 \mathrm{~cm}$. Le $\mathrm{pH}$ maximum est 7,7 à $420 \mathrm{~cm}$.

Le taux de matière organique est de $8,33 \%$ en $A 1$ et reste supérieur à $1,00 \%$ jusqu'à $100 \mathrm{~cm}$. Les horizons inférieurs ont un taux moyen de $0,73 \pm 0,19 \%$. Le $\mathrm{C} / \mathrm{N}$ est de 13,0 en $A 1$. L'horizon II BC (200280) est le seul à renfermer du $\mathrm{CaCO}_{3}$.

L'indice d'instabilité is moyen est de $2,52 \pm 0,19$ entre 40 et $380 \mathrm{~cm}$, pour 1,4 en surface $(10-20 \mathrm{~cm})$. Is présente 2 maximums $(2,73)$ entre 80 et $100 \mathrm{~cm}(\mathrm{Bt})$ et entre 280 et $300 \mathrm{~cm}$ (III Bt).

\section{Analyse du système racinaire}

\section{Profil racinaire}

Selon un cylindre vertical fictif, situé à $1 \mathrm{~m}$ autour de l'arbre, l'estimation du nombre total moyen de racines s'élève à 2049 , soit 1191,411 et 447 racines de 0 à 60 $\mathrm{cm}, 60$ à 120 et 120 à $400 \mathrm{~cm}$ de profondeur (tableau III). Les valeurs de densité

Tableau III. Système racinaire des chênes pédonculés : nombre total moyen de racines observées à $1 \mathrm{~m}$ de l'arbre, à l'intersection d'un cylindre fictif.

\begin{tabular}{cccccc}
\hline $\begin{array}{c}\text { Profondeur } \\
(\mathrm{cm})\end{array}$ & $\begin{array}{c}\text { Classe a } \\
(1-5 \mathrm{~mm})\end{array}$ & $\begin{array}{c}\text { Classe b } \\
(5-20 \mathrm{~mm})\end{array}$ & $\begin{array}{c}\text { Classe } \mathrm{c} \\
(20-50 \mathrm{~mm})\end{array}$ & $\begin{array}{c}\text { Classed } \\
(>50 \mathrm{~mm})\end{array}$ & Total \\
\hline $0-60$ & 963 & 168 & 33 & 27,3 & 1191,3 \\
$60-120$ & 324 & 60 & 27 & 0 & 411 \\
$120-400$ & 405 & 42,3 & 0 & 0 & 447,3 \\
Total & 1692 & 270,3 & 60 & 27 & 2049,3 \\
\hline
\end{tabular}


racinaire subdivisent la distribution des racines en 2 parties principales (fig 5 et fig 1C) :

- un système de surface, de 0 à $60 \mathrm{~cm}$ de profondeur, qui comporte une masse racinaire très importante caractérisée par une densité racinaire élevée représentant $85 \%$ de la densité totale;

- un système profond, de 60 à $400 \mathrm{~cm}$ de profondeur, qui comprend une masse racinaire encore assez dense de $60-120 \mathrm{~cm}$ et un ensemble de racines dispersées très profondément jusqu'à $400 \mathrm{~cm}$.

Le maximum de densité racinaire se situe à l'intérieur du système de surface, entre 20 et $30 \mathrm{~cm}(35,5 \%)$.
La couche très superficielle, de 0-10 $\mathrm{cm}$ de profondeur, n'est prospectée que par des racines fines de classe a ou b (diamètre $1-5$ et $5-20 \mathrm{~mm}$ ). Toutes les classes de diamètre $a, b, c$ et $d$, sont représentées entre 10 et $60 \mathrm{~cm}$. Les grosses racines d (supérieures à $50 \mathrm{~mm}$ ) disparaîssent après $60 \mathrm{~cm}$. Les racines c $(20-50 \mathrm{~mm})$ subsistent jusqu'à $120 \mathrm{~cm}$. Les racines fines (classes $a$ et $b$ ) colonisent les matériaux pédologiques jusqu'à $4 \mathrm{~m}$ et certaines d'entre elles prospectent les fissures du karst; elles ont été dégagées jusqu'à 4,5 m.

Grâce au test $t$ (Student), on a pu calculer que, pour les valeurs de densité totales, les parties $0-60$ et $60-120 \mathrm{~cm}$ sont statisti-

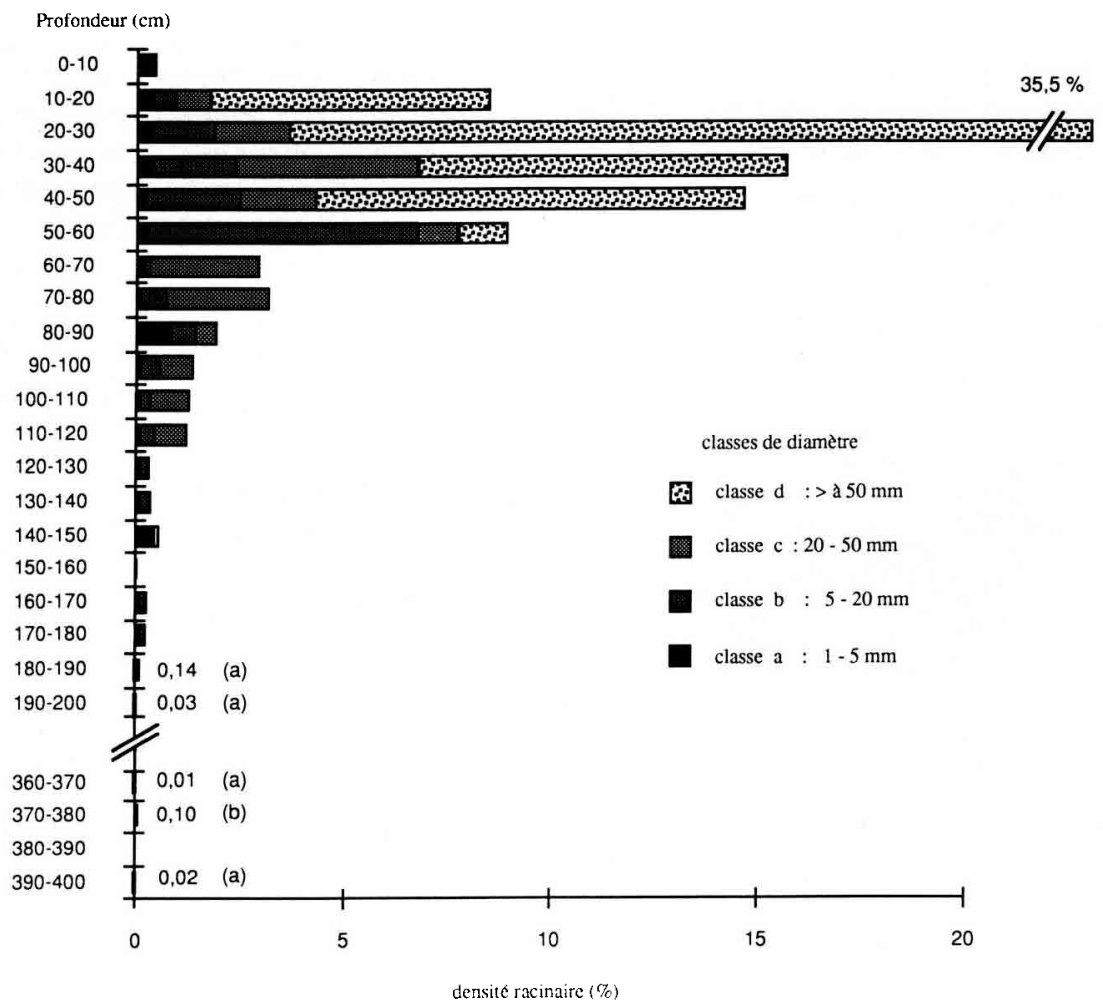

Fig 5. Distribution moyenne des racines en fonction de la profondeur et par classe de diamètre. La densité racinaire est exprimée en $\%$ de la densité totale. Les valeurs de densité racinaire et les classes de diamètre concernées sont indiquées lorsqu'elles sont trop faibles pour être représentées. 
quement différentes à $98,9 \%$, les parties $0-60$ et $120-400 \mathrm{~cm}$ le sont à $100 \%$, de même que les parties $60-120$ et 120 $400 \mathrm{~cm}$.

\section{Ramification, extension et angles racinaires}

À partir du tronc, un nombre moyen de 7,4 contreforts par arbre forment une première ossature du système de surface. Écartés les uns des autres selon des angles de 30$60^{\circ}$, ils se ramifient en 2 racines dans un rayon moyen de $0,50 \mathrm{~m}$, soit 14 racines primaires latérales qui vont coloniser le sol de façon extensive à plus de $20 \mathrm{~m}$ de l'arbre, sans s'enfoncer au-delà de $60 \mathrm{~cm}$ (profondeur moyenne $36 \mathrm{~cm} \pm 12,4$ ). Elles se ramifient particulièrement dans leur mètre distal. Un schéma de l'organisation racinaire est représentée spatialement selon un repère orthogonal dans la figure 6 .

Au-dessous des contreforts, prend naissance une deuxième couronne de racines primaires latérales (14 en moyenne) dont l'extension ne dépasse pas $3 \mathrm{~m}$. Ces dernières se ramifient et émettent des pivots secondaires dans un rayon de 2,5 $\mathrm{m}$ autour du tronc.

Au-dessous de la deuxième couronne de racines, le tronc émet encore des pivots primaires qui vont se développer en profondeur. L'émission des pivots primaires et secondaires se produit à une profondeur moyenne de $55 \mathrm{~cm}$. Après un parcours de 2-3 $\mathrm{m}$, les racines du système superficiel conservent une direction de croissance dans la mesure où elles ne rencontrent pas d'obstacle.

Les pivots se ramifient eux-même en l'absence de toute contrainte en 2 ou 3 racines de plus faible diamètre et de même inclinaison, entre 95 et $150 \mathrm{~cm}$ de profondeur (profondeur moyenne de $110 \mathrm{~cm}$ ). Compte tenu des extensions et des densités racinaires observées, le volume de sol prospecté de manière intensive par le système racinaire des chênes étudiés, âgés

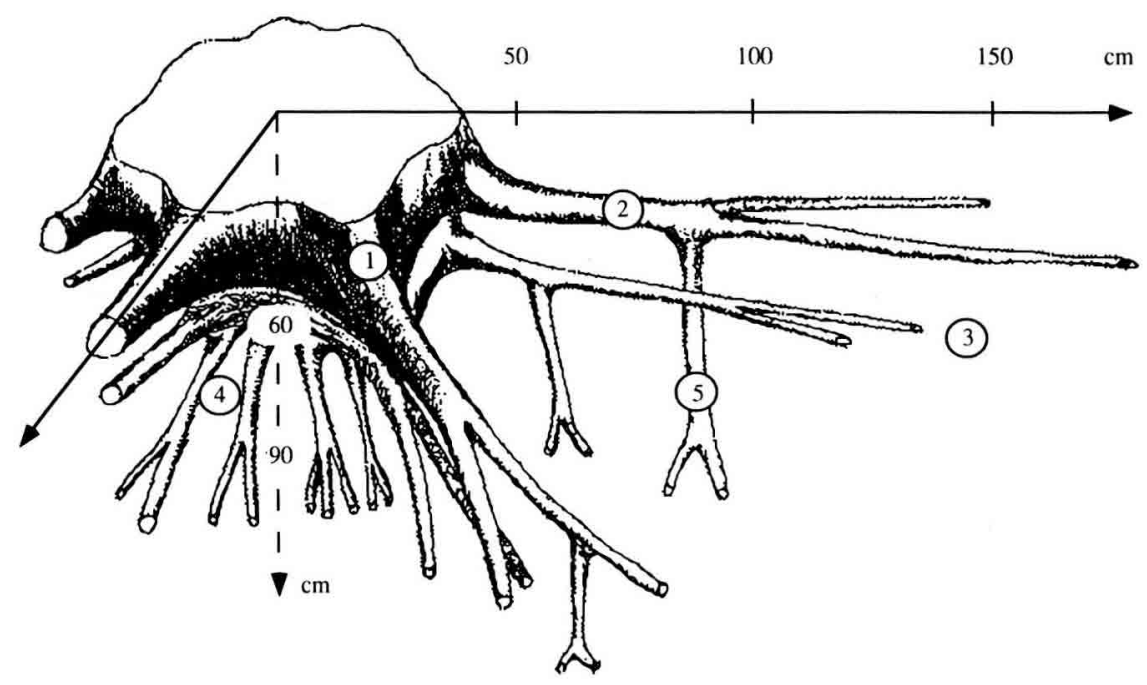

Fig 6. Organisation du système racinaire du chêne pédonculé : contreforts (1), grosses racines primaires latérales (2, diamètre $>50 \mathrm{~mm}$ ), deuxième couronne de racines (3), pivots primaires (4) et secondaires (5). 
de 145 ans en moyenne, serait de $17 \mathrm{~m}^{3}$. Au-delà de ce volume, les systèmes racinaires de surface et de profondeur peuvent coloniser de façon extensive des volumes de sol de 50 et $750 \mathrm{~m}^{3}$.

L'inclinaison des racines par rapport à la verticale est schématisée sur la figure 7 . Les mesures groupées par niveau de 10 $\mathrm{cm}$ ont conduit à établir des angles moyens qui décroissent avec la profondeur. Dans le système de surface, l'angle moyen est de $83^{\circ}$; de 60 à $110 \mathrm{~cm}$, le système racinaire comportant des racinaires et des départs de pivots prend une inclinaison moyenne de $57^{\circ}$; au-delà de $110 \mathrm{~cm}$, les pivots et leurs émissions se développent avec un angle moyen de $35^{\circ}$.

Ces 3 ensembles de racines $(0-60,60$ 110 et $<110 \mathrm{~cm}$ ) sont statistiquement différents à $99,8 \%$ au minimum, pour les valeurs moyennes de l'inclinaison par niveau de $10 \mathrm{~cm}$. Les écarts types sont de l'ordre de 7,3 dans les 60 premiers $\mathrm{cm}$, de 25,2 entre 60 et $110 \mathrm{~cm}$ et 23,2 dans la partie inférieure à $110 \mathrm{~cm}$.

\section{DISCUSSION}

Les paramètres racinaires que nous avons quantifiés, sont synthétisés dans le tableau IV. Ils apparaissent en accord avec les caractéristiques des systèmes racinaires des chênes pédonculés décrits dans la littérature (Sika, 1963; Kostler et al, 1968; Belgrand, 1983). Le système de surface représente $80-85 \%$ de la densité racinaire totale mesurée sur le profil à $1 \mathrm{~m}$ de l'arbre et ces valeurs sont conformes aux mesures de biomasse racinaire de Büsgen et Münch (1927).

La première couronne du système de surface (racines structurales de premier ordre) prospectent latéralement le sol de manière extensive. L'extension latérale dépend d'une part de l'âge de l'arbre (Ber-

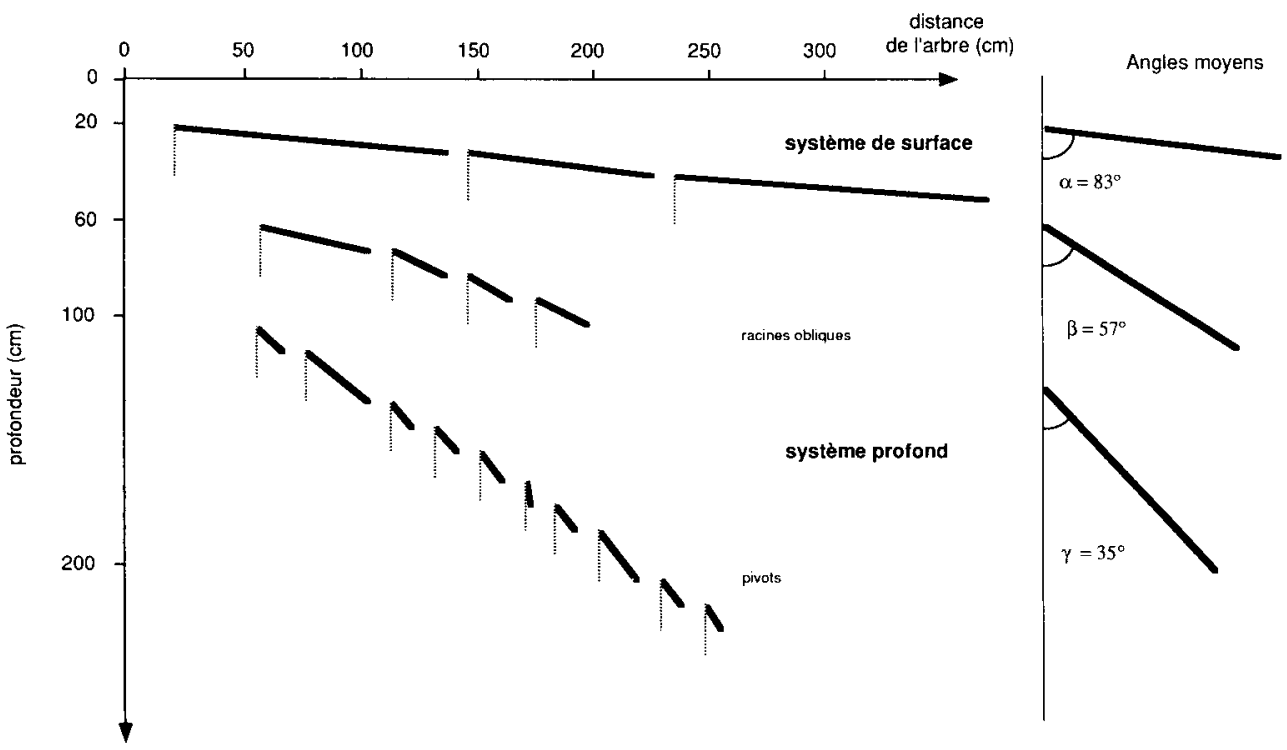

Fig 7. Représentation schématique de l'écart moyen des racines par rapport à la verticale, par niveau de $10 \mathrm{~cm}$ et pour chaque partie du système racinaire $(\alpha, \beta, \gamma)$. 
Tableau IV. Paramètres racinaires moyens pour les différentes portions du système racinaire du chêne pédonculé. Le diamètre moyen et la densité racinaire sont mesurés à $1 \mathrm{~m}$ de l'arbre.

\begin{tabular}{|c|c|c|c|c|c|}
\hline $\begin{array}{l}\text { Profondeur } \\
\quad(\mathrm{cm})\end{array}$ & $\begin{array}{l}\text { Diamètre } \\
(\mathrm{mm})\end{array}$ & $\begin{array}{l}\text { Extension } \\
\quad(m)\end{array}$ & Inclinaison & $\begin{array}{c}\text { Densité } \\
(\%)\end{array}$ & Qualification \\
\hline \multicolumn{6}{|c|}{ Système racinaire de surface } \\
\hline $\begin{array}{c}0-10 \\
10-60\end{array}$ & $\begin{array}{r}3,4 \\
9,0 \\
90,0\end{array}$ & $\begin{array}{r}3,0 \\
3,0 \\
20,0\end{array}$ & $\begin{array}{c}- \\
80-85^{\circ} \\
-\end{array}$ & $\begin{array}{r}0,5 \\
85,5 \\
60,5\end{array}$ & $\begin{array}{l}\text { très superficiel } \\
\text { intensif } \\
\text { extensif }\end{array}$ \\
\hline \multicolumn{6}{|c|}{ Système racinaire profond } \\
\hline $\begin{array}{c}60-120 \\
120-400\end{array}$ & $\begin{array}{l}6,3 \\
3,7\end{array}$ & $\begin{array}{l}2,5 \\
2,0\end{array}$ & $\begin{array}{l}55-60^{\circ} \\
30-40^{\circ}\end{array}$ & $\begin{array}{r}12,0 \\
3,0\end{array}$ & $\begin{array}{l}\text { intensif } \\
\text { extensif }\end{array}$ \\
\hline
\end{tabular}

ben, 1968; Ruark et al, 1982) et d'autre part de la concurrence avec les racines des autres arbres, donc de la densité de peuplement (McMinn, 1963; GagnaireMichard et Guizerix, 1976). Le rôle de ces racines est d'accroître la stabilité de l'arbre, mais aussi le volume de sol prospecté et la masse disponible des éléments nutritifs (Reynolds, 1987). Ces racines qui se développent en longueur sont parfois assimilées à des racines de famine en station pauvre (De Champs et Dufour, 1976), la richesse trophique favorisant surtout le développement en diamètre et en profondeur. Or, la station ayant un niveau trophique élevé, les racines observées ne peuvent être considérées comme des racines de famine, mais comme celles caractéristiques d'un développement optimal. Ce développement dépasse de $12 \mathrm{~m}$ l'extension maximale du houppier, souvent prise comme référence pour estimer la prospection racinaire latérale. Cet écart s'explique par les différences de l'impact de la compétition aérienne et souterraine sur le développement, respectivement, des houppiers et des systèmes racinaires. Certaines essences forestières méditerranéennes sur sol caillouteux, ont un sys- tème racinaire traçant à plus de $30 \mathrm{~m}$ du tronc (Callot et al, 1983).

La deuxième couronne (inférieure) du système de surface stoppe sa croissance avant $3 \mathrm{~m}$ de distance de l'arbre, ce qui s'accorde avec les observations de Belgrand (1983). Dans ce rayon de 2,5-3 m de l'arbre, l'enracinement est considéré comme intensif (Pritchett, 1979).

Le système racinaire profond peut être subdivisé en 2 parties. Celle qui s'étend entre 60 et $120 \mathrm{~cm}$ comporte à la fois des racines obliques et des pivots. Les grosses racines (classe d) sont absentes et le diamètre moyen est de $6,3 \mathrm{~mm}$. L'angle moyen par rapport à la verticale est de 55$60^{\circ}$. Les valeurs de cet angle varient de manière importante car ce niveau regroupe des racines obliques $\left(75^{\circ}\right)$ et des pivots $\left(40^{\circ}\right)$. Ces racines s'étendent peu au-delà de $2,5 \mathrm{~m}$ de l'arbre. Un des paramètres retenu pour caractériser le développement vertical des pivots est leur profondeur de ramification, les racines se ramifiant au contact d'un obstacle (Riedacker, 1978; Riedacker et Dexheimer, 1982; Lucot, 1990) où elles émettent des racines latérales. Lorsque les pivots subissent une contrainte et produisent des racines laté- 
rales non verticales, celles-ci apparaîssent sur le profil et induisent un accroissement de la densité racinaire. Ce phénomène est ici très discret et perceptible seulement au niveau du système profond. Les ramifications observées (2-3 par pivot) ne sont pas dues à une contrainte, mais représentent le développement normal de ces racines.

La partie inférieure à $120 \mathrm{~cm}$ (système profond) est prospectée par les pivots, dans un rayon de $2 \mathrm{~m}$ autour de l'arbre. L'angle moyen évolue de $40-30^{\circ}$ vers la profondeur. Cet angle est très lié aux conditions pédologiques et une contrainte se traduit par une direction plus horizontale. Le diamètre moyen est de $3,7 \mathrm{~mm}$ et le diamètre de chacune de ces racines diminue très peu entre 120 et $400 \mathrm{~cm}$ de profondeur. Ces racines fines sont très verticales et ne recoupent le profil (cylindre vertical à $1 \mathrm{~m}$ de l'arbre) qu'en cas de contraintes. Avec leur cortège de racines très fines, elles prospectent le sol en profondeur.

L'architecture du système racinaire est très sensible aux conditions pédologiques, comme la texture, la densité apparente, la stabilité structurale, la perméabilité et l'aération (Weller, 1965; Köstler et al, 1968; Levy, 1968; Berben, 1973; Callot et al, 1983; Sutton et Tinus, 1983; Fitter, 1987). La comparaison des variations de porosité et de densité racinaire en fonction de la profondeur (figs 4 et 5) rend compte de l'évolution rigoureusement parallèle des 2 paramètres $\left(r^{2}=0,91\right)$. Dans les stations étudiées, les matériaux prospectés par les racines offrent des textures limonoargileuses, puis argilo-limoneuses, séparées par une légère discontinuité limonoargilo-sableuse qui ne constitue nullement un obstacle au développement du système profond. Les densités apparentes de 1,41 à 1,56 mesurées tout au long des profils n'entravent pas la prospection racinaire. Les valeurs observées ne dépassent effec- tivement pas le seuil de 1,55 proposé par Bowen (1981). De même, les indices d'instabilité structurale de 1,40-2,73 rencontrés dans les matériaux ne gênent pas les développements racinaires, qui sont influencés par des indices plus élevés d'après Berben (1973), Zimmerman et Kardos (1981). L'ensemble des matériaux prospectés par les racines est perméable et aéré, comme en témoignent les critères de structuration, de porosité et de couleur notés à tous les niveaux : l'organisation des horizons profonds résulte d'une intense dynamique de gonflement - retrait des argiles sous l'effet d'alternances répétées d'humectation - dessiccation, caractéristique des horizons argiliques (Bruckert et Bekkary, 1992); l'organisation des horizons de surface est très marquée par les structurations d'origine biologique (Bruckert et Selino, 1978; Selino et al, 1978; Tajosky et al, 1991). Les couleurs brunes uniformes sont celles des complexes argilohumiques dans lesquels le fer, à l'état oxydé, demeure fortement associé aux argiles (Ghabru et al, 1990).

Dans les conditions pédologiques très favorables rencontrées dans les stations étudiées, le volume de sol prospecté de façon intensive $\left(17 \mathrm{~m}^{3}\right)$ et extensive (800 m3) apparaît considérable. Étant donné que les conditions d'alimentation quantitative et qualitative d'un arbre dépendent de ce volume (Schlots et al, 1956; Cottreau, 1972; De Champs et Dufour, 1976; Ezenwa, 1985), on peut se demander quelles sont les parts respectives assurées par les enracinements intensif et extensif dans la nutrition de l'arbre et sa production. Des recherches seront à développer concernant les besoins en eau et en oxygène des racines selon leur situation dans l'architecture observée et concernant les profils hydriques développés saisonnièrement in situ.

En conclusion, les paramètres racinaires mesurés ici en conditions édaphi- 
ques optimales serviront de modèle de référence dans l'étude des enracinements en conditions contraignantes, en particulier limités en profondeur par un obstacle physique ou chimique (Bruckert, 1989), comme la pierrosité (Lucot, 1990), la compacité ou l'hydromorphie. L'architecture, les déformations et les adaptations racinaires développées en présence d'obstacles seront comparées aux références définies en conditions non contraignantes dans le but d'estimer les volumes rhizofonctionnels, dénominateur commun reliant les propriétés des sols à la production des arbres.

\section{REMERCIEMENTS}

Les auteurs remercient la commune de Fontain et l'Office national des forêts pour avoir autorisé les travaux de terrain malgré leur ampleur ainsi que MM F Groell, A Laurent et D Paget pour leur aide technique.

\section{RÉFÉRENCES}

Belgrand M (1983) Comportement de jeunes plants feuillus (chêne pédonculé, chêne rouge, chêne sessile, hêtre) sur substrat ennoyé. Adaptations racinaires. Thèse INAPG, $188 \mathrm{p}$

Berben JC (1968) Problèmes d'enracinement en sols sableux. Bull Soc R For Belg 5, 233-249

Berben JC (1973) Influence de la densité du sol et des précipitations sur la croissance et le développement radiculaire de quelques essences forestières. Bull Soc R For Belg 10 , 377-401

Bowen HD (1981) Alleviating mechanical impedance. In: Modifying the root environment to reduce crop stress. ASAE monograph $n^{\circ} 4$, Michigan USA, 18-57

Bruckert S (1989) Désignation et classement des sols agricoles d'après des critères de situation et d'organisation : application aux terres francs-comtoises du domaine climati- que tempéré semi-continental. Agronomie 9, 353-351

Bruckert S, Bekkary M (1992) Formation des horizons diagnostiques argiliques et de fragipan en fonction de la perméabilité des roches. Can J Soil Sci 72, 69-88

Bruckert S, Selino D (1978) Mise en évidence de l'origine biologique et chimique des structures micro-agrégées foisonnantes des sols bruns ocreux. Pédologie, Gand, XXVIII (1), 46-59

Büsgen, Münch (1927) Bau und Leben unserer Waldbäume. Jena. In: Köstler et al (1968)

Callot G, Maertens C et Salsac $L$ (1983) Les interactions sol-racine, incidence sur la nutrition minérale. INRA, $325 \mathrm{p}$

De Champs J, Dufour J (1976) Étude de l'enracinement en place sur jeunes douglas. AFOCEL, 163-215

Cottreau R (1972) Le travail du sol et la croissance des semis de pin maritime. AFOCEL, 91-139

Coutts MP (1989) Factors affecting the direction of growth of tree roots. Ann Sci For 46, 277287

CPCS (1967) Classification des sols. ENSA Grignon, $97 \mathrm{p}$

Duchaufour P (1991) Pédologie. Masson, Paris, $189 \mathrm{p}$

Ezenwa MIS (1985) Effects of some soil physical properties on growth of Pinus caribaea in northern Guinea savanna area of Nigeria. Research Paper, Savanna For Res St Ser, Samane, Zairia, 49-56

FAO-UNESCO (1989) Quideline for soil profile description. $125 \mathrm{p}$

Fitter AH (1987) An architectural approach to the comparative ecology of plant root systems. New Phytol 106, 61-77

Forristall F, Gessel SP (1955) Soil properties related to forest cover type and productivity on the Lee forest, Snohomish County, Washington. Soil Sci Soc Proc America, 19, 384-389

Gagnaire-Michard J, Guizerix J (1976) Étude non destructive des racines d'un épicéa en forêt. AFOCEL 76, 147-159

Ghabru SK, Saint-Arnaud RJ, Mermu AR (1990) Association of DCB-extractable iron with $\mathrm{mi}$ nerals in coarse soil clays. Soil Sci 149 (2), $112-120$ 
Henderson R, Ford ED, Renshaw E, Deans JD (1983) Morphology of the structural root system of Sitka spruce. 1. analysis and quantitative description. Forestry 56 (2), 121-135

Köstler JN, Brückner E, Bibelriether $H$ (1968) Die Wurzeln der Waldbäume. Verlag Paul Parey, Hamburg, $284 \mathrm{p}$

Levy G (1968) Importance du sol pour l'enracinement de Picea excelsa et de Pinus sylvestris. Ann Sci For 25 (3), 157-188

Lucot $E$ (1990) Première approche relative à l'interaction enracinement-pierrosité. Proposition d'un indice d'obstacle appliqué aux sols forestiers jurassiens. DEA de Pédologie, Besançon, $57 \mathrm{p}$

McKeague JA, Wang C, Topp GC (1982) Estimating satured hydraulic conductivity from soil morphology. Soil Sci Soc Am J 46, 1239-1244

McMinn RG (1963) Caracteristics of douglas fir root systems. Can J Bot 41, 105-122

Martens $M$ (1975) Le fonctionnement des racines. $C R$ gpe étude racines 25 octobre 1974 (Nancy) et 22 octobre 1975 (Grenoble), 2, 200-205

Mourey JM (1979) Les chênes de la vallée de l'Ognon. Rapport ENITEF, $284 \mathrm{p}$

Pritchett WL (1979) Properties and managment of forest soils. John Wiley and Sons, New York, $491 \mathrm{p}$

Rameau JC, Mansion D, Dume G (1989) Flore forestière française. 1. Plaines et collines. IDF, $1784 \mathrm{p}$

Reynolds ERC (1987) Development of the root crown in some conifers. Plant Soil 98, 397-405

Riedacker A (1978) Étude de la déviation des racines horizontales ou obliques issues de boutures de peuplier qui rencontrent un obstacle : application pour la conception de conteneurs. Ann Sci For 35 (1), 1-18
Riedacker A, Dexheimer J (1982) Modifications expérimentales de la morphogénèse et des géotropismes dans le système racinaire de jeunes chênes. Can J Bot 60, 765-778

Ruark GA, Mader DL, Tattar TA (1982) The influence of soil compaction and aeration on the root growth and vigour of tree - a literature review. Part 1. Arboric J 6, 251-265

Schlots FE, Lloyd WJ, Deardoroff CE (1956) Some soil characteristics which affect root penetration and timber site quality of Douglas fir in western Washington. Soil Sci Soc Proc Am 20, 101-105

Selino D, Proth J, Bruckert S, Kilbertus G (1978) Sols acides structurés en agrégats : analyse d'un mode d'agrégation d'origine biologique. $103^{\circ}$ Congrès Nat Soc Sav Nancy Sci fasc 209-225

Sika $A$ (1963) Le système racinaire de quelques espèces feuillues sur sols limoneux. Prace Vulm CSSR. In: Kostler et al (1968)

Sutton RF, Tinus RW (1983) Root and root system terminology. For Sci 23 (4)

Tajosky K, Villemin G, Toutain F (1991) Microstructural and ultrastructural change of the Oak leaf litter consumed by Millepede Glomer hexasticha (diplopode). Rev Ecol Biol Sol (sous presse)

Vartanian N (1974) Diversité morphologique du système racinaire en relation avec l'humidité édaphique. $\mathrm{C} R$ gpe étude racines 25 octobre 1974 (Nancy) et 22 octobre 1975 (Grenoble), 2, 166-179

Weller F (1965) Die Ausbreitung der Pflanzenwurzeln im Boden in Abhängigkeit von genetischen und ökologischen Faktoren. Verlag Eugen Ulmer Stuttgart, $123 p$

Zimmerman RP, Kardos LT (1981) Effect of bulk density on root growth. Soil Sci $91,280-288$ 